\title{
Low- $\omega 3$ Fatty Acid and Soy Protein Attenuate Alcohol-Induced Fatty Liver and Injury by Regulating the Opposing Lipid Oxidation and Lipogenic Signaling Pathways
}

\author{
Karina Reyes-Gordillo, Ruchi Shah, Ravi Varatharajalu, Mamatha Garige, \\ Leslie C. Leckey, and M. Raj Lakshman
}

Lipid Research Laboratory, VA Medical Center and Department of Biochemistry and Molecular Medicine, The George Washington University, Washington, DC, USA

Correspondence should be addressed to M. Raj Lakshman; raj.lakshman@va.gov

Received 5 August 2016; Revised 14 October 2016; Accepted 15 November 2016

Academic Editor: Tullia Maraldi

Copyright (C) 2016 Karina Reyes-Gordillo et al. This is an open access article distributed under the Creative Commons Attribution License, which permits unrestricted use, distribution, and reproduction in any medium, provided the original work is properly cited.

Chronic ethanol-induced downregulation of peroxisome proliferator-activated receptor gamma coactivator 1-alpha (PGCl $\alpha)$ and upregulation of peroxisome proliferator-activated receptor gamma coactivator 1-beta $(\mathrm{PGCl} \beta)$ affect hepatic lipid oxidation and lipogenesis, respectively, leading to fatty liver injury. Low- $\omega 3$ fatty acid (Low- $\omega 3$ FA) that primarily regulates PGC1 $\alpha$ and soy protein (SP) that seems to have its major regulatory effect on $\mathrm{PGCl} \beta$ were evaluated for their protective effects against ethanol-induced hepatosteatosis in rats fed with Lieber-deCarli control or ethanol liquid diets with high or low $\omega 3$ FA fish oil and soy protein. Low$\omega 3 \mathrm{FA}$ and SP opposed the actions of chronic ethanol by reducing serum and liver lipids with concomitant decreased fatty liver. They also prevented the downregulation of hepatic Sirtuin 1 (SIRT1) and PGC1 $\alpha$ and their target fatty acid oxidation pathway genes and attenuated the upregulation of hepatic PGC1 $\beta$ and sterol regulatory element-binding protein 1c (SREBP1c) and their target lipogenic pathway genes via the phosphorylation of $5^{\prime}$ adenosine monophosphate-activated protein kinase (AMPK). Thus, these two novel modulators attenuate ethanol-induced hepatosteatosis and consequent liver injury potentially by regulating the two opposing lipid oxidation and lipogenic pathways.

\section{Introduction}

Alcohol liver disease is a major cause of morbidity and mortality, affecting millions world-wide [1]. Long-term exposure of ethanol causes fatty liver disease or hepatosteatosis [2], which further leads to steatohepatitis, fibrosis, and finally cirrhosis that may result in death [3]. Hepatosteatosis is characterized by the accumulation of lipids, triglyceride and cholesterol, due to an imbalance between hepatic lipid degradation and synthesis, leading to an enlarged fatty liver [3]. Studies have shown that alcohol causes the following: (i) increased mobilization of adipose fat into the liver, due to increased adipose lipoprotein lipase, (ii) decreased fat oxidation due to downregulation of fatty acid oxidation genes, (iii) increased fat synthesis due to upregulation of lipogenic genes, and (iv) impaired synthesis of apolipoprotein
$B$ and secretion of very low density lipoprotein (VLDL), the major lipoprotein for the export of hepatic lipids to peripheral tissues [4].

Transcriptional coactivators peroxisome proliferator receptor coactivator 1 alpha (PGC1 $\alpha$ ) and peroxisome proliferator receptor coactivator 1 beta $(\mathrm{PGCl} \beta)$ as well as sterol regulatory element-binding proteins (SREBPs) play vital roles in regulating the lipid oxidizing and lipogenic genes and thereby control the progression of hepatosteatosis and the consequent onset of fibrosis and other forms of liver injury $[5,6]$. Peroxisome proliferator-activated receptors (PPARs) are members of the nuclear hormone receptor super family that are ligand-dependent transcription factors. There are three isotypes, namely, PPAR $\alpha, \operatorname{PPAR} \beta$, and PPAR $\gamma$. Whereas PPAR $\alpha$ is expressed in all tissues controlling the fatty acid oxidation pathway genes, PPAR $\gamma$ is primarily expressed in 
adipose tissue and the liver, regulating the lipogenic pathway genes. PPAR $\beta$ is found in many tissues although mainly in gut, kidney, and heart [7-9]. It is linked to colon cancer [10] but has not been well studied. PGC1 $\alpha$ regulates lipid oxidation pathway genes via $\operatorname{PPAR} \alpha$ and $\mathrm{PGCl} \beta$ regulates lipogenic pathway genes via the sterol regulatory elementbinding proteins SREB1a, SREB1c, and SREBP2 [11]. SREB1c predominantly regulates fatty acid biosynthesis while SREBla and SREBP2 control cholesterol synthesis [3]. AMP activated protein kinase (AMPK) is known to be activated by phosphorylation to form phosphorylated AMPK (pAMPK), which, in turn, phosphorylates and inactivates acetyl CoA carboxylase (ACC) and the rate-limiting enzyme of lipogenesis $[4,12,13]$. PGC1 $\alpha$ is controlled by silence regulator gene 1 (SIRT1), the eukaryotic equivalent of SIR2 gene in prokaryotes, and histone acetyltransferases (HAT) [14]. SIRT1 activates PGC1 $\alpha$ by deacetylation while HAT inactivates PGC1 $\alpha$ by acetylation [15]. On the other hand, SIRT1 destabilizes SREBP1c by deacetylation while HAT stabilizes SREBP1c by acetylation [16]. PGC1 $\beta$ is upregulated by dietary saturated fat and coactivates SREBP1c and liver X receptor (LXR) families of transcription factors leading to increased lipogenesis, lipoprotein transport, and VLDL secretion [17, 18]. Therefore, any modulator that can either activate $\mathrm{PGCl} \alpha$ via the interplay between SIRT1 and histone acetyltransferase (HAT) or inactivate PGC1 $\beta /$ SREBP1c should be beneficial in preventing alcoholic hepatosteatosis and consequent liver injury.

Omega-3/6 fatty acids are polyunsaturated fatty acids (PUFA) obtained from fish and plant sources. The most common omega-3 PUFA are eicosapentaenoic acid (EPA), docosahexaenoic acid (DHA), and alpha-linolenic acid (ALA). Whereas algae and oils from fish such as salmon, mackerel, and herring are rich in EPA and DHA, ALA is found in vegetable oils such as canola, flax seed oil, soybean oil, and nuts such as walnuts [19]. Soy proteins (SP) are found in soybean legume containing all 8 essential amino acids and very low saturated fat [20]. In recent times, both omega-3 PUFA and SP have received increased attention due to their beneficial effects against cardiovascular disease, obesity, type 2 diabetes, and certain cancers, among others [19, 21, 22]. Low omega 3 fatty acids (low- $\omega 3 \mathrm{FA}$ ) are known to have lipid lowering effects in humans [23] while SP lowers plasma and liver cholesterol and triglycerides in both animals and humans [24]. Studies have shown that SP prevents hyperinsulinemia and reduces the expression of LXRs and SREBP1c mRNAs in obese Zucker rat model [25-27]. However, the molecular mechanisms by which these dietary modulators can control the two transcriptional coactivators are yet to be explored. In this study, we demonstrate the novel actions of low- $\omega 3 \mathrm{FA}$ and SP in inhibiting alcoholic hepatosteatosis by regulating two opposing vital pathway genes of lipid degradation and synthesis via PGC1 $\alpha$ and $\mathrm{PGCl} \beta$, respectively. Therefore, low- $\omega 3$ FA and SP are potentially potent dietary modulators that seem to have these profound lipid lowering properties involving lipid catabolic and anabolic pathways. Moreover, low- $\omega 3$ FA and SP stimulate AMPK phosphorylation and block ethanol-induced increased lipogenesis. Thus, this may be the first time a systematic approach is made to alleviate alcoholic hepatosteatosis by the combined effects of novel natural modulators that promise to intervene with both lipid oxidizing and lipogenic pathways.

\section{Material and Methods}

2.1. Animals. Wild-type (WT) female Wistar rats $(\sim 150 \mathrm{~g}$ body weight) from Charles River, Wilmington, MA, were housed in pairs per cage in plastic cages, in a temperaturecontrolled room, at $25^{\circ} \mathrm{C}$ with 12-hours light-dark cycle. All animals were fed a pelleted commercial diet (Purina Rodent Chow, number 500, TMI Nutrition, St. Louis, MO) during the first week of acclimation period after arrival. Experiments were performed according to the approved institutional animal care and use committee protocol. Female rats were randomly divided into 4 groups of 5 rats each and were pairfed Lieber-DeCarli control or ethanol $(\mathrm{EtOH})$ liquid diets (36\% total fat calories) with high- $\omega 3 \mathrm{FA}$ (14.1\% of calories as $\omega 3 \mathrm{FA})$ or low- $\omega 3 \mathrm{FA}(2.7 \%$ of calories as $\omega 3 \mathrm{FA})$ fish oil or EtOH with SP for 4 weeks.

2.2. Diets. The diets are isocaloric and their formulations are according to the modified method of Lieber and DeCarli [28] with the recommended normal nutrients, vitamins, and minerals according to AIN-93 diet [29]. Thus, 36\% of the total energy of ethanol diet is from fat, $20 \%$ from protein, $36 \%$ from $\mathrm{EtOH}$, and the rest from the carbohydrate. The corresponding isocaloric control diet has isoenergetic amounts of dextrinmaltose in place of EtOH. EtOH concentration in the liquid diet was gradually increased starting at $1 \%$ level on day 1 and reaching the $5 \%$ level over a 7 -day period to allow the animals to adapt to EtOH in the diet. These diets are supplemented with $120 \mathrm{IU}$ of tocopherol/L and $200 \mathrm{mg} / \mathrm{L}$ of tertiary-butyl hydroquinone as antioxidants as per AIN-93 diet recommendations $[28,29]$.

2.3. Lipid and Lipoprotein Analysis. Blood samples were collected and centrifuged at $3100 \mathrm{rpm}$ using a Beckman J6M (Beckman Coulter, Indianapolis, IN) for $10 \mathrm{~min}$ at $4^{\circ} \mathrm{C}$. Separated serum, plasma, and liver samples were frozen at $-80^{\circ} \mathrm{C}$ until assayed. Liver lipids and high density lipoproteins (HDL) were extracted as previously described [30, 31]. Cholesterol was analyzed using Sigma diagnostic kit number 352 (Sigma-Aldrich, St. Louis, MO) according to the method of Allain et al. [32] and triglycerides were analyzed using Sigma diagnostic kit number 339 (Sigma-Aldrich, St. Louis, MO) according to the method of McGowan et al. [33]. All protein concentration determinations were done according to Bradford method [34] with bovine serum albumin (BSA) as the standard.

\subsection{Isolation of Plasma HDL and Its Labeling with $\left[{ }^{3} \mathrm{H}\right]$ Cholesteryl Oleate. HDL was isolated from various pooled groups of rat plasma according to Gidez et al. [30]. Protein concentration was determined colorimetrically using bovine serum albumin (BSA) as a standard [34]. HDL cholesterol content was measured according to Zlatkis and Zak [35]. HDL labeling with $\left[{ }^{3} \mathrm{H}\right]$ cholesteryl oleate was performed according to Basu et al. [36], and the specific activity is expressed as dpm/mg HDL cholesterol.}


2.5. Quantification of Hepatosteatosis by Oil Red O. Livers from various experimental groups were cut into small pieces and washed immediately with ice cold PBS and mounted on optimum cutting temperature (OCT) embedding compound in peel-a-way embedding molds (Electron Microscope Sciences, Hatfield, PA). Liver tissues were cryosectioned and stained with oil red $\mathrm{O}$ to measure accumulation of lipid using an automated histometric system (Image-Pro Plus 6.1, Media Cybernetics, Bethesda, MD) as described previously [37]. The data are expressed as average oil red O percentage area of lipid staining. Values are means \pm SEM.

2.6. RNA Isolation and Real-Time RT-PCR. The total RNA was isolated from each liver using the Tri-Reagent (Molecular Research Center, Cincinnati, $\mathrm{OH}$ ) as manufacturer's instructions. Isolated total RNA was reverse transcribed by in vitro transcription as described by the manufacturer (Invitrogen, Carlsbad, CA). Quantitative real-time PCR was performed using a Bio-Rad iCycler using the SYBR green PCR mix (Bio-Rad, Hercules, CA). Typical real-time PCR reaction mixture included same amount of cDNA templates from RT, $10 \mathrm{pM}$ of each primers, $10 \mu \mathrm{M}$ of dNTPs, $3 \mathrm{mM}$ of $\mathrm{MgCl}_{2}$, 10x buffer, and $2 \mu$ of high fidelity Taq DNA polymerase in a reaction volume of $50 \mu \mathrm{L}$ with $0.1 \mathrm{x}$ SYBR Green I. The PCR conditions were $3 \mathrm{~min}$ at $95^{\circ} \mathrm{C}$ followed by 40 cycles at $95^{\circ} \mathrm{C}$ for 30 seconds, $55^{\circ} \mathrm{C}$ for 30 seconds, and $72^{\circ} \mathrm{C}$ for $1 \mathrm{~min}$. Each primer pair was first tested by regular PCR to be highly effective and specific for amplification. $\beta$-Actin was used as the standard housekeeping gene. Ratios of specific mRNA and actin mRNA expression levels were calculated by subtracting the threshold cycle number $(\mathrm{Ct})$ of the target gene from the $\mathrm{Ct}$ of actin and raising 2 to the power of this difference. Ct values were defined as the number of PCR cycles at which the fluorescent signal during the PCR reaches a fixed threshold. Target gene expressions were expressed relative to $\beta$-actin expression. The various primer pairs for indicated rat genes and transcription factors are listed in Supplemental Table 1 in Supplementary Material available online at http://dx.doi.org/10.1155/2016/1840513.

2.7. Western Blot Analysis. Liver extracts from each experimental group were diluted into SDS-PAGE sample buffer [50 mM Tris (pH 6.8), 2\% SDS, $10 \%$ glycerol, $15 \mathrm{mM} \mathrm{2-}$ mercaptoethanol, and $0.25 \%$ bromophenol blue] and electrophoretically resolved in Novex (Life Technologies, San Diego, CA) 4-20\% denaturing polyacrylamide gels. Proteins are electrophoretically transferred to PVDF membrane and processed for immunodetection using the corresponding polyclonal primary antibodies for each of the above factors. After thorough washing, the primary antibody was detected with horse radish peroxidase conjugated secondary antibody specific to IgG of the respective primary antibody. Protein bands were visualized by chemiluminescence and quantified using FluorChem Imager (Alpha Innotech, CA). The nuclear extracts from each group were analyzed for the level of SIRT1, $\mathrm{PGCl} \alpha$, and $\mathrm{PGCl} \beta$ and the mature form of SREBPlc in the respective groups using the respective specific antibodies, while total protein extracts were analyzed for the levels of ACC, c-Met, AMPK, and pAMPK using respective specific antibodies. To determine the levels of acetylated-PGCl $\alpha$, the liver nuclear extract from each group was initially immunoprecipitated with anti-PGCl $\alpha$ followed by immunoblotting with acetylated lysine antibody. The polyclonal antibodies for all the above transcription factors were purchased from Santa Cruz Biotechnology (Santa Cruz, CA), Cayman Chemicals (Ann Arbor, MI), and UpState Cell Signaling Solutions (Lake Placid, NY). The specificity of each antibody was verified before use for the above analyses.

2.8. Immunoprecipitation Analysis. Immunoprecipitation was performed as previously described [38]. To determine the levels of acetylated-PGCl $\alpha$, the liver nuclear extract from each group was initially immunoprecipitated with anti-PGCl $\alpha$ (Abcam, Cambridge, MA), followed by immunoblotting with acetylated lysine antibody (Cell Signaling Technology, Danvers, MA).

2.9. Statistical Analysis. Experimental data were statistically analyzed, employing the paired and unpaired " $t$ " tests on the control and the experimental values. The appropriate data were analyzed by one-way or two-way analysis of variance (ANOVA) at $p<0.05$ followed by Tukey contrast to evaluate the true correlation between various parameters.

\section{Results}

3.1. Effects of Chronic Ethanol, Low- $\omega 3$ FA, or SP on Serum and Liver Lipids and Hepatic Lipid Score. Serum cholesterol (Figure 1(a)) and triglycerides (Figure 1(b)) were significantly increased in EtOH group by 1.8 -fold $(p<0.05)$ and 1.2-fold $(p<0.05)$, respectively, compared to control. Similarly, total liver cholesterol (Figure 1(c)) and triglycerides (Figure 1(d)) were also markedly increased in EtOH group by 3.9-fold $(p<0.05)$ and 4.1-fold $(p<0.05)$, respectively, compared to control. In contrast, dietary low- $\omega 3$ FA or SP feeding to EtOHfed groups significantly decreased serum and liver cholesterol and triglycerides to the level closer to that of the control group. Furthermore, the hepatic accumulation of lipids as measured by oil red $\mathrm{O}$ staining is markedly increased in $\mathrm{EtOH}$ group by 7.5 -fold $(p<0.001)$ as compared to the control. This effect is significantly reduced after dietary administration of low- $\omega 3 \mathrm{FA}$ and SP in the EtOH-fed group by $93 \%(p<0.05)$ and $45 \%(p<0.05)$, respectively (Figure 1(e)).

3.2. Effects of Low- $\omega 3 F A$ and SP on EtOH-Mediated Alterations in the Lipid Oxidation Pathway. Chronic EtOH leads to a significant decrease in fatty acid oxidation (48.7 \pm $5.8 \mathrm{nmoles} / \mathrm{g} / \mathrm{h}, p<0.05)$ as compared to control $(100 \pm$ $8.6 \mathrm{nmoles} / \mathrm{g} / \mathrm{h}$ ). We further investigated whether the mechanisms of action of low- $\omega 3 \mathrm{FA}$ and SP on EtOH-induced decrease in fatty acid oxidation are mediated via the regulation of the transcriptional coactivator PGCl $\alpha$, SIRT1, and the downstream pathway. Figure 2(a) showed that low- $\omega 3$ FA and SP treatment restored chronic EtOH-mediated 32\% $(p<$ $0.05)$ downregulation in SIRT1 mRNA by $85 \%(p<0.05)$ and $80 \%(p<0.05)$, respectively, as compared to $\mathrm{EtOH}$ group. EtOH also significantly downregulated PGCl $\alpha$ mRNA 


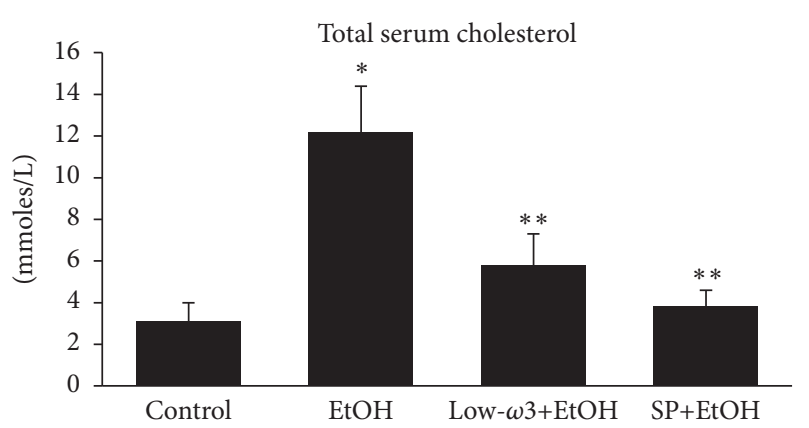

(a)

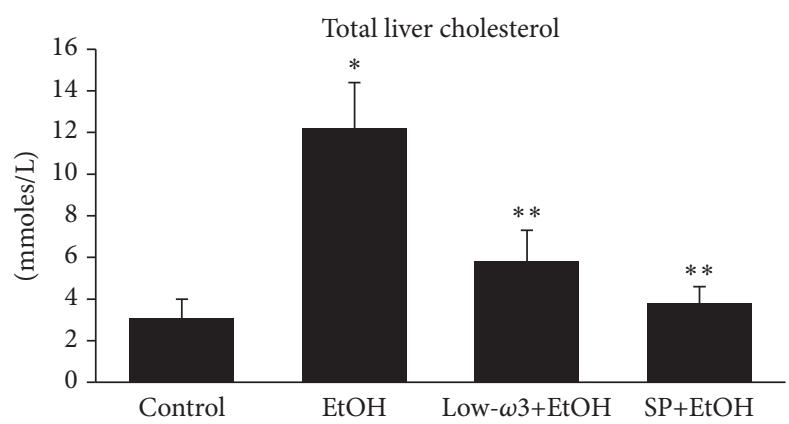

(c)

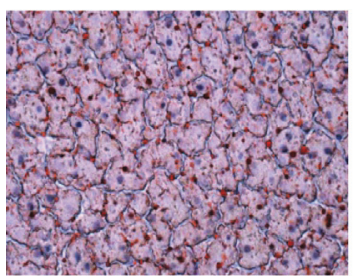

Control

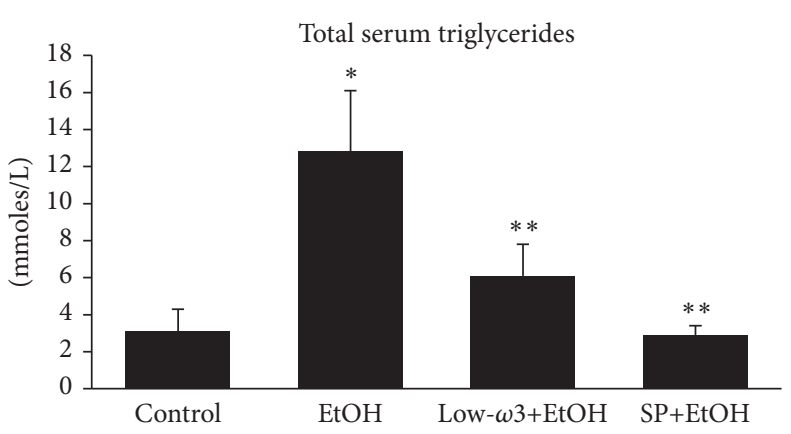

(b)

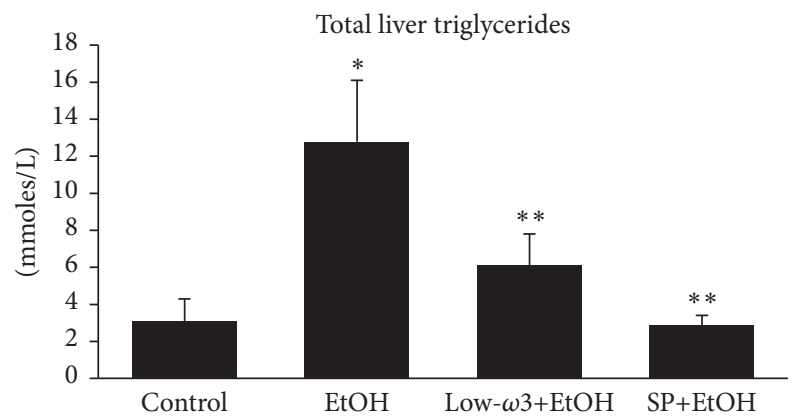

(d)

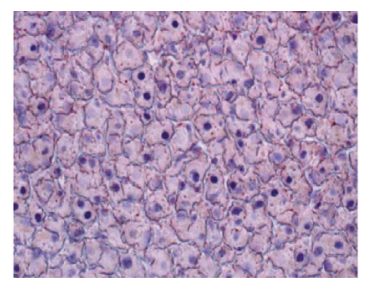

Low- $\omega 3+\mathrm{EtOH}$

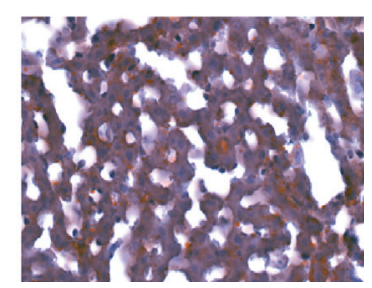

$\mathrm{SP}+\mathrm{EtOH}$

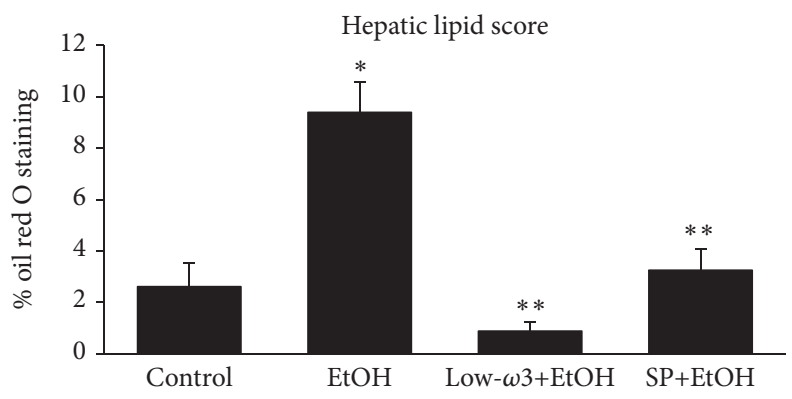

(e)

FIgURE 1: Influence of EtOH, low- $\omega 3$ FA, and SP on (a) total serum cholesterol, (b) serum triglycerides, (c) total liver cholesterol, and (d) liver triglycerides. Each value is mean \pm SD of 3 samples/group. (e) shows the representative medium-power (20x) photomicrographs of liver sections stained with oil red $\mathrm{O}$ as described in Section 2 and the plot of the mean hepatic lipid scores of all samples in each group \pm SE of 3 samples/group. ${ }^{*} p<0.05$ versus control; ${ }^{* *} p<0.05$ versus EtOH.

by $40 \%(p<0.05)$ that was restored to 1.5 -fold $(p<0.05)$ and 2 -fold $(p<0.05)$ over the control level by low- $\omega 3$ FA and SP treatment, respectively (Figure 2(b)). Additionally, CPT1 mRNA was also markedly downregulated by chronic EtOH $(24 \%, p<0.05)$ which was restored to 1.5 -fold $(p<0.05)$ over the control level by these dietary modulators (Figure 2(c)). Similarly, chronic EtOH markedly decreased the nuclear protein expression of SIRT1 and PGC1 $\alpha$ by $38 \%(p<0.05)$ and $35 \%(p<0.05)$, respectively, which was restored over the control levels by low- $\omega 3$ FA and SP treatment (Figures 2(d) and 2(e)). PPAR $\alpha$, a ligand-activated transcription factor, involved in the regulation of hepatic fatty acid oxidation [39], was also evaluated. EtOH significantly decreased PPAR $\alpha$ protein levels by $50 \%(p<0.05)$ that was restored by $1.8-$ fold $(p<0.05)$ and 1.6-fold $(p<0.05)$ by low- $03 \mathrm{FA}$ and SP treatment, respectively (see supplementary materials, Figure S1). Thus, low- $\omega 3$ FA and SP are effective modulators in correcting the decreased fatty acid oxidation caused by 


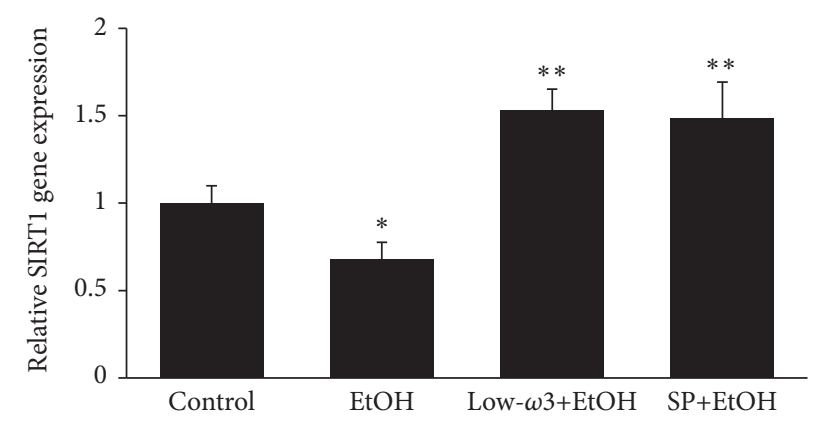

(a)

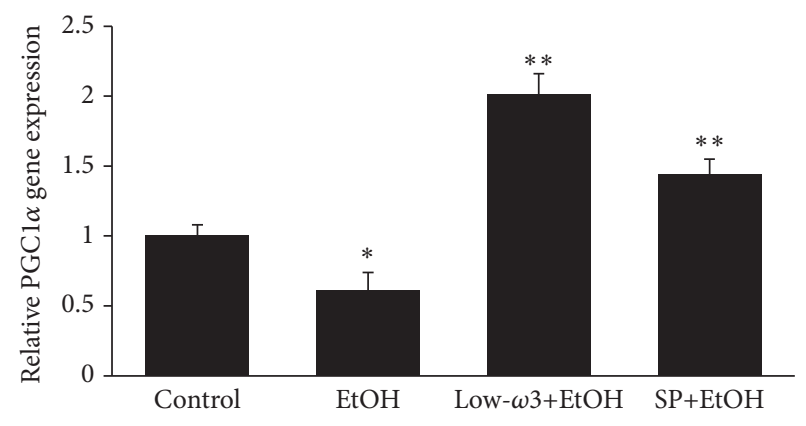

(b)

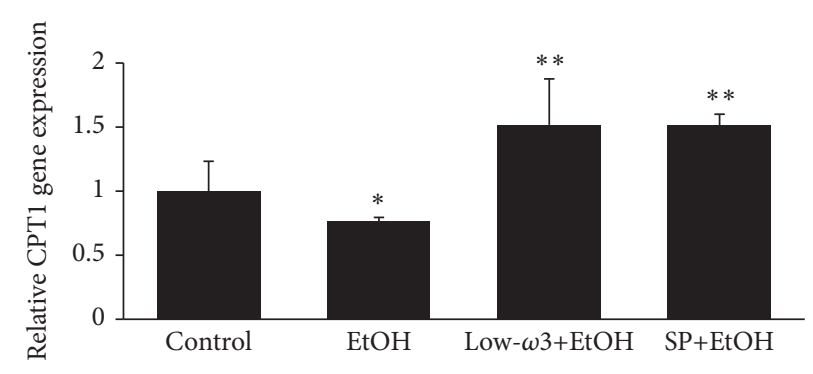

(c)

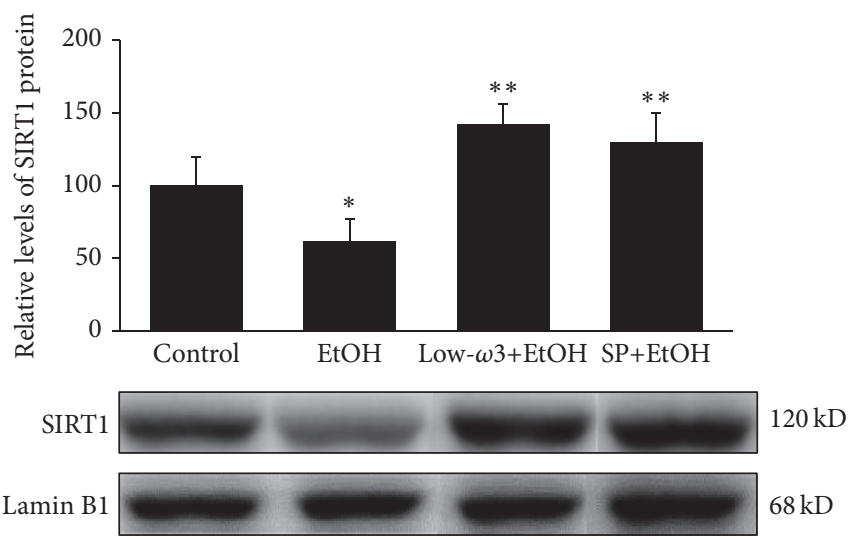

(d)

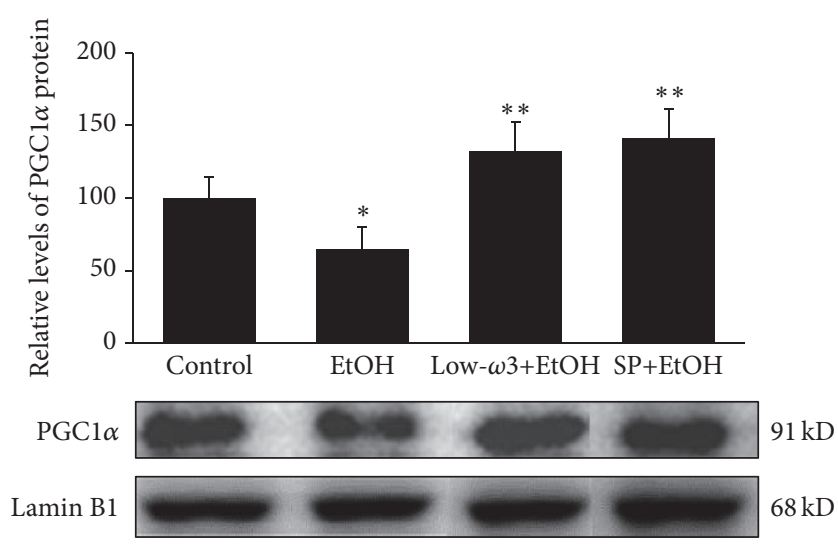

(e)

Figure 2: Influence of EtOH, low- $\omega 3$ FA, and SP on lipid oxidation pathway. Total RNA from each animal was reverse transcribed and used in the qRT-PCR analysis using gene specific primers as described in Section 2 for (a) SIRT1, (b) PGC1 $\alpha$, and (c) CPT1. Each gene was normalized to $\beta$-actin mRNA. Nuclear protein was extracted from each animal and used for Western Blot analysis using specific antibodies as described in Section 2 for (d) SIRT1 and (e) PGCl $\alpha$. Values are means of triplicate experiments \pm SD of 3 samples/group and were corrected for difference in loading after reprobing with an antibody to Lamin B1. ${ }^{*} p<0.05$ versus control; ${ }^{* *} p<0.05$ versus EtOH.

chronic EtOH via the regulation of SIRT1, PGC1 $\alpha$, CPT1, and $\operatorname{PPAR} \alpha$.

In order to test whether the action of low- $\omega 3$ FA and SP on hepatic lipid catabolism was mediated through the active or inactive forms of PGC1 $\alpha$ via the modulation of SIRT1, we determined the level of acetylated PGC1 $\alpha$ in the liver tissue of various groups. Figure 3 shows that chronic EtOH increased the hepatic acetylated (inactive) form of PGC1 $\alpha$ by $40 \%(p<0.05)$ because of EtOH-mediated decrease in SIRT1 by $38 \%(p<0.05)$ as compared to the control (Figure $2(d)$ ), thereby accounting for decreased fatty acid oxidation. In contrast, low- $\omega 3 \mathrm{FA}$ and SP decreased the inactive form of PGC1 $\alpha$ by $37 \%$ and $25 \%$, respectively, as compared to $\mathrm{EtOH}$ group (Figure 3) via the upregulation of SIRT1 (Figures 2(a) and $2(\mathrm{~d})$ ), thereby accounting for restoring the decreased fatty acid caused by chronic EtOH to the control level. Thus, low- $\omega 3$ FA and SP may lower alcoholic hepatosteatosis by augmenting the relative levels of active form of PGC1 $\alpha$; that in turn effectively restored hepatic lipid catabolism that is impaired by chronic alcohol exposure.

\subsection{Effects of Low-w3FA and SP on Chronic EtOH-Mediated} Alterations in the Lipogenic Pathway. Figure 4(a) shows that chronic EtOH markedly upregulated PGCl $\beta$ mRNA level by $52 \%(p<0.05)$ as compared to the control, and low- $\omega 3 \mathrm{FA}$ and SP downregulated the EtOH effect by $61 \%(p<0.05)$ and $55 \%(p<0.05)$, respectively. Similarly, Figure 4(b) shows a marked 50\% $(p<0.05)$ upregulation in SREBP1c mRNA that 


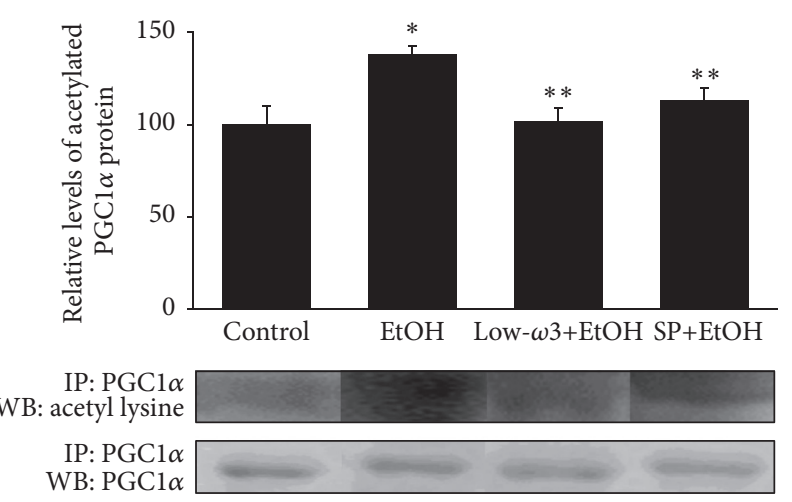

FIGURE 3: Effect of EtOH, low- $\omega 3$ FA, and SP on hepatic acetylatedPGCl $\alpha$ levels. Western blot analysis was performed with an acetyl lysine-specific antibody of a nuclear protein extract immunoprecipitated (IP) with an antibody to PGC1 $\alpha$ as described in Section 2. Values are means of triplicate experiments \pm SD of 3 samples/group. ${ }^{*} p<0.05$ versus control; ${ }^{* *} p<0.05$ versus EtOH.

was reduced to $30 \%$ and $50 \%(p<0.02)$ of the control value by low- $\omega 3 \mathrm{FA}$ and SP treatment, respectively. Chronic EtOH also markedly upregulated the mRNA expression levels of ACC, which regulates fatty acid synthesis by 2 -fold $(p<0.05)$ and this was significantly suppressed by $50 \%(p<0.05)$ in the low- $\omega 3$ FA-EtOH group and by $70 \%(p<0.05)$ in SP-EtOH group (Figure $4(\mathrm{c})$ ). In contrast, as shown in Figure 4(d), the mRNA expression levels of c-Met were significantly downregulated by 35\% $(p<0.05)$ after chronic EtOH administration, and low- $\omega 3$ FA and SP treatment significantly restored $\mathrm{EtOH}$-induced downregulation of c-Met mRNA level to 86\% ( $p<0.05)$ and 95\% ( $p<0.05)$, of the control value, respectively. These results were confirmed by measuring the nuclear or total protein expression of the above genes relative to those of the corresponding subcellular marker proteins. Figure 4(e) shows that low- $\omega 3$ FA and SP fed rats showed suppressed EtOH-mediated increase $(60 \%, p<$ $0.05)$ in the relative nuclear expression of $\mathrm{PGCl} \beta$ by $68 \%(p<$ $0.05)$ and $63 \%(p<0.05)$, respectively. Similarly, as shown in Figures $4(\mathrm{f})$ and $4(\mathrm{~g})$, the relative nuclear protein expressions of SREBP1c and ACC were also markedly increased in chronic $\mathrm{EtOH}$ group by 30\% $(p<0.05)$ and 50\% $(p<0.05)$, respectively, compared to the control group. Administration of dietary low- $\omega 3 \mathrm{FA}$ and SP reversed these EtOH-mediated effects by decreasing SREBP1c protein expression by $50 \%$ $(p<0.05)$ and $56 \%(p<0.05)$, respectively (Figure 4(f)), and ACC protein expression by $85 \%(p<0.05)$ and $60 \%$ $(p<0.05)$, respectively (Figure $4(\mathrm{~g}))$. On the other hand, $c$-Met expression was decreased in the EtOH group by $25 \%$ $(p<0.05)$, which were restored in low- $\omega 3 \mathrm{FA}$ and SP groups by $35 \%(p<0.05)$ and $45 \%(p<0.05)$, respectively, as compared to the EtOH group (Figure 4(h)).

Since chronic EtOH increases hepatic ACC activity and lipogenesis by decreasing the phosphorylation of AMPK (pAMPK), a known inhibitor of ACC, we tested whether low$\omega 3 \mathrm{FA}$ or SP can counteract these effects of chronic EtOH by modulating the phosphorylation status of AMPK. As shown in Figures 5(a) and 5(b), although the level of total AMPK was unaffected in all groups, low- $\omega 3 \mathrm{FA}$ and SP restored the hepatic level of pAMPK that was decreased by $50 \%(p<0.05)$ in EtOH group. This increase in pAMPK could also account for decreased ACC activity and lipogenesis after low- $\omega 3$ FA or SP treatment.

These findings are consistent with the ability of low$\omega 3 \mathrm{FA}$ or SP to (i) inhibit chronic EtOH-induced increase in lipogenic pathway genes and (ii) restore ethanol-mediated decreased intracellular transport of hepatic triglycerides to the blood compartment due to impaired VLDL assembly and secretion. This would lead to the low- $\omega 3$ FA or SP-mediated reduction in fatty liver caused by chronic alcohol abuse.

\section{Discussion}

Our results show that low- $\omega 3$ FA and SP exert their hypolipidemic action by upregulating primarily the lipid oxidizing genes via SIRT1 and PGCl $\alpha$ signaling pathway that are suppressed by chronic ethanol and downregulating the lipogenic pathway genes predominantly via the PGC1 $\beta$ and SREBP1c signaling pathway. Our data also support the alternative possibility that low- $\omega 3 \mathrm{FA}$ and SP could prevent alcoholinduced activation of ACC activity by phosphorylating it via pAMPK.

SIRT1 is an NAD-dependent deacetylase (histone deacetylase (HDAC)) that has been linked to many beneficial effects of cellular processes including gene silencing, insulin resistance, glucose homeostasis, fatty acid metabolism, and aging, while HAT catalyses the opposite reaction [40]. Thus, SIRT1 activates PGCl $\alpha$ by deacetylation while HAT inactivates PGCl $\alpha$ by acetylation. On the other hand, SIRT1 destabilizes SREBP1c by deacetylation while HAT stabilizes SREBP1c by acetylation. You et al. [16] and Lieber et al. [41] have elegantly shown that both long chain and medium chain saturated fatty acids in the diet restore the expressions of SIRT1 and PGCl $\alpha$ that are downregulated by long chain polyunsaturated fatty acids (PUFA) in chronic ethanol-fed animals. However, PPAR $\gamma$ was unaffected by chronic ethanol. Previously, Fischer et al. [42] have shown in mice that ethanol leads to PPAR $\alpha$ dysfunction resulting in impaired fatty acid oxidation and consequent onset of fatty liver that is overcome by a PPAR $\alpha$ agonist. Similarly, other studies [43, 44] have shown that alcohol-mediated fatty liver and injury are prevented by PPAR $\gamma$ agonist presumably by activating cMet and blocking alcohol-mediated induction of TNF $\alpha$. We recently showed that compared to high fish oil control liquid diet, feeding of the same high fish oil liquid diet containing $5 \%(w / v)$ ethanol for 8 weeks significantly downregulated hepatic SIRT1, and PGCl $\alpha$ with the concomitant decreased hepatic rate of fatty acid oxidation [37]. Nanji et al. [45], Ronis et al. [46], and Song et al. [47] have demonstrated that saturated fatty acids protect against chronic alcohol-induced liver injury as compared to high levels of polyunsaturated fatty acids. In addition, Huang et al. [48] demonstrated that low levels of omega 3 polyunsaturated fatty acids, mainly docosahexaenoic acid, suppressed ethanol-induced hepatic steatosis. Similarly, Wada et al. [49] also demonstrated that low levels of fish oil fed prior to ethanol administration 


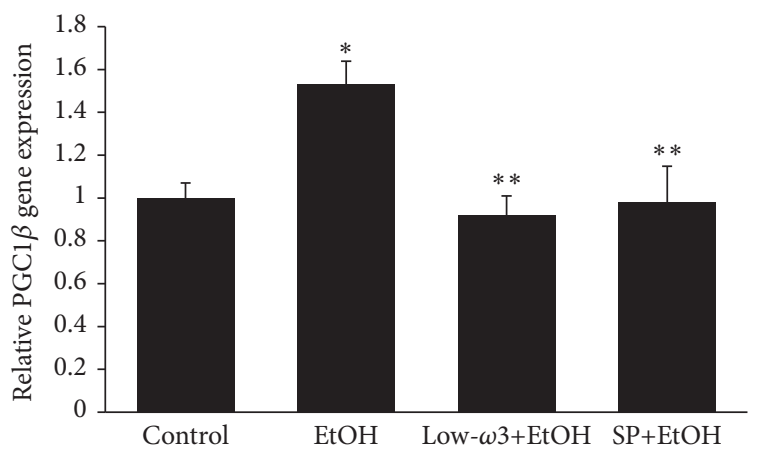

(a)

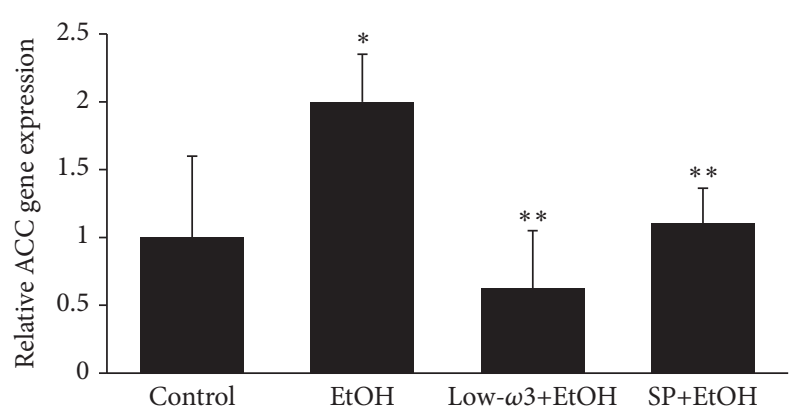

(c)

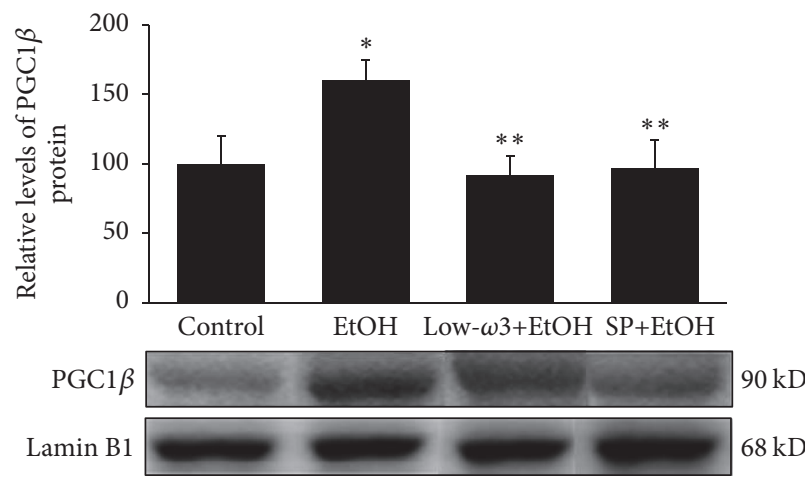

(e)

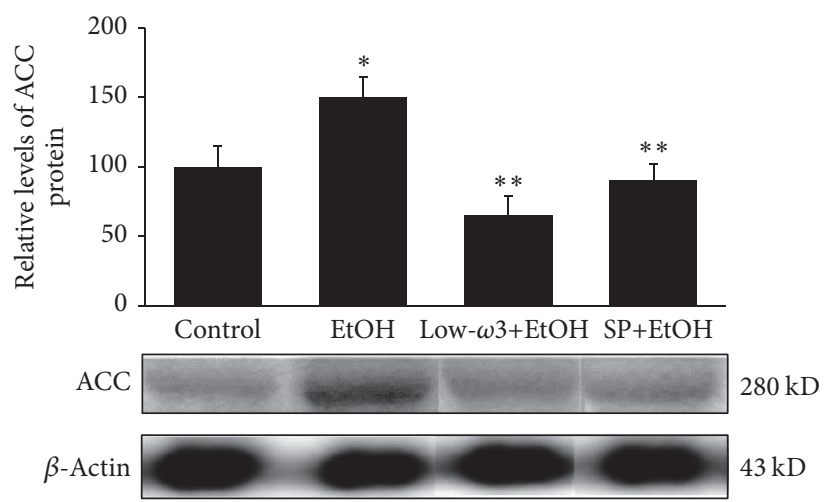

(g)

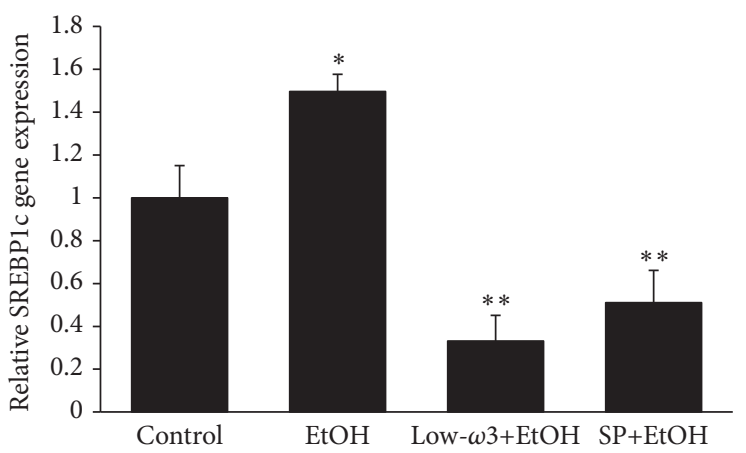

(b)

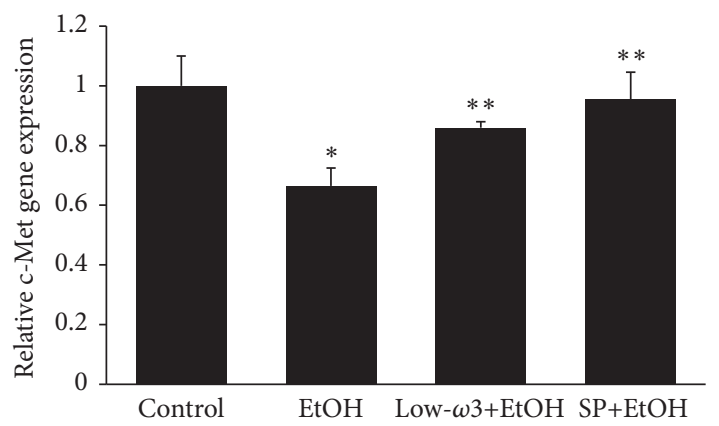

(d)

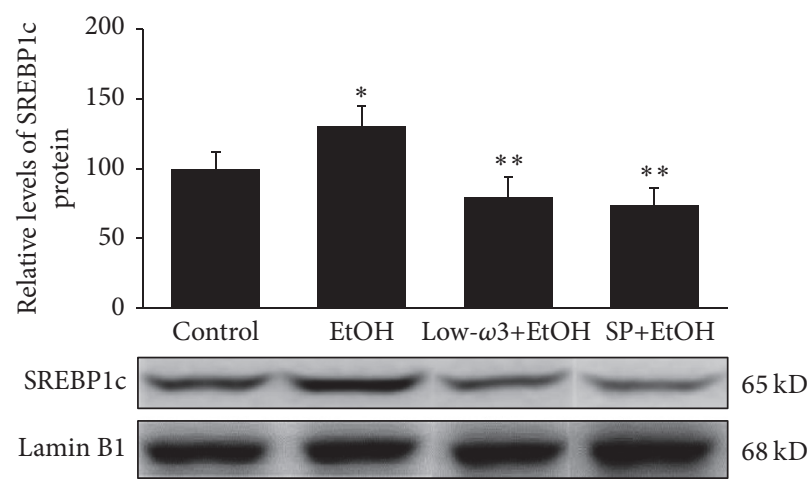

(f)
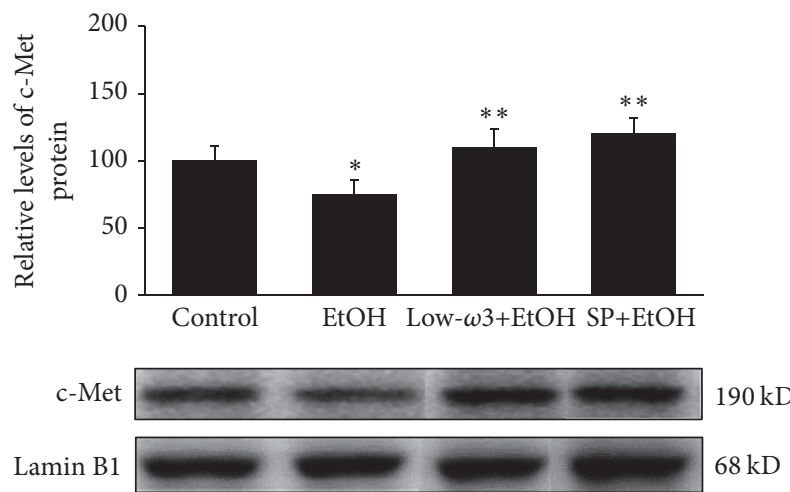

(h)

FIGURE 4: Influence of EtOH, low- $\omega 3 \mathrm{FA}$, and SP on lipogenic pathway. Total RNA from each animal was reverse transcribed and used in the qRT-PCR analysis using gene specific primers as described in Section 2 for (a) PGC1 $\beta$, (b) SREBP1c, (c) ACC, and (d) c-Met. Each gene was normalized to $\beta$-actin mRNA. Nuclear or total protein was extracted from each animal and used for Western Blot analysis using specific antibodies as described in Section 2 for (e) PGC1 $\beta$, (f) SREBP1c, (g) ACC, and (h) c-Met. Values are means of triplicate experiments \pm SD of 3 samples/group and were corrected for difference in loading after reprobing with an antibody to Lamin B1 or $\beta$-actin for nuclear or total protein, respectively. ${ }^{*} p<0.05$ versus control; ${ }^{* *} p<0.05$ versus EtOH. 


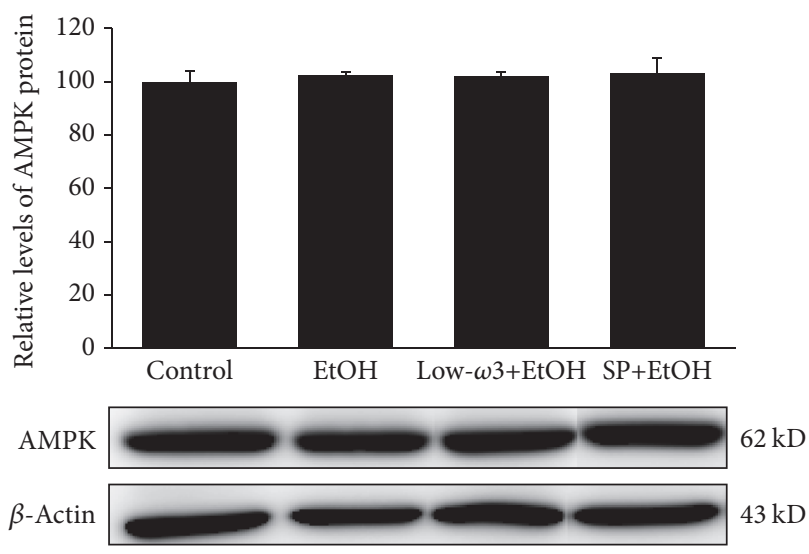

(a)

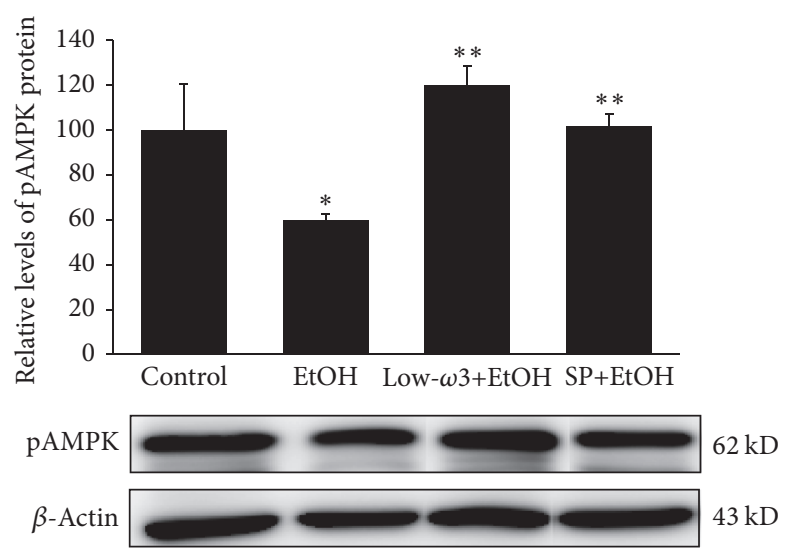

(b)

FIGURE 5: Effect of EtOH, low- $\omega 3$ FA, and SP on hepatic (a) AMPK and (b) pAMPK was determined. Total protein was extracted from each animal and used for Western Blot analysis using specific antibodies as described in Section 2. Values are means of triplicate experiments \pm $\mathrm{SD}$ of 3 samples/group and were corrected for difference in loading after reprobing with an antibody to $\beta$-actin. ${ }^{*} p<0.05$ versus control; ${ }^{* *} p<0.05$ versus EtOH.

prevent acute ethanol-induced fatty liver in mice. In agreement with these studies, the present study shows that dietary low level of $\omega 3 \mathrm{FA}(2.7 \%)$, but not high level of $\omega 3 \mathrm{FA}$ (14.1\%), restores the expression of SIRT1 and PGC1 $\alpha$ that are downregulated by chronic ethanol. Our results also show that chronic alcohol exposure upregulates PGC1 $\beta$, ACC, c-Met, and SREBP1c. Activation of SREBP1c by ethanol feeding in rats had been already associated with increased expression of hepatic lipogenic genes as well as the accumulation of triglyceride in the livers [50]. However, Ki et al. [51], Lu et al. [52], and Zeng et al. [53] demonstrated that SREBP-1c-mediated lipogenesis pathway was not affected by ethanol or even suppressed after chronic ethanol intake in rats. While the inability of these studies to show chronic ethanol-mediated upregulation of SREBP-1c-mediated lipogenic pathway may be due to different dietary fat compositions compared to the present study, we consistently find that the upregulation of this pathway with the high- $\omega 3$ FA diet was markedly attenuated by low- $\omega 3$ FA diet. Thus, it is important to point out that different dietary conditions, particularly the amount and the type fat in the diet, do affect SREBP activation pathway in different ways.

Low- $\omega 3$ FA have an inherent property of attenuating chronic alcohol-mediated hepatosteatosis by upregulating PGC1 $\alpha$ and downstream lipid degradation pathways while SP downregulates PGC1 $\beta$, SREBP1, and downstream lipid synthetic pathways and by controlling the active/inactive forms of AMPK. Recently, Phillipson et al. demonstrated the lipid lowering effects of fish oil rich in $\omega 3$ FA in humans [23]. However, chronic ethanol-induced liver damage in rats fed a high fat diet (36\% fat calories) was exacerbated [45, 46, 54-56] with polyunsaturated FA from either vegetable oil ( $\omega 6$ family) or fish oil ( $\omega 3$ family) as evidenced by increased serum aspartate aminotransferase and alanine aminotransferase as well as by histopathology. In this study, fish oil constituted $36 \%$ of the total calories in the diet, which amounted to $14.1 \%$ of the total dietary calories as $\omega 3 \mathrm{FA}$. In contrast, we showed [57] that the inclusion of only $2.7 \%$ of total dietary calories as $\omega 3 \mathrm{FA}$ resulted in lower plasma and liver lipids in chronic alcohol-fed animals. Furthermore, the same low level of dietary $\omega 3 \mathrm{FA}$ restored the decreased ApoE content in HDL. Thus, a low level of $\omega 3$ FA has beneficial effects [58$60]$, whereas a significant increase in $\omega 3 \mathrm{FA}$ seems to have a detrimental effect on the liver $[45,46,54-56]$. It is possible that increased ethanol consumption in the intragastric model could have also caused the deleterious effects when PUFArich diet was fed [55]. Significantly, PUFA-containing lecithin diet was shown to prevent alcohol-induced hepatic fibrosis in baboons [61]. We showed [57] that low- $\omega 3$ FA caused decreased VLDL production and serum lipids resulting in lipid-deficient ApoE, which can be easily sialylated and be associated with HDL. This would be consistent with the effects of low- $\omega 3$ FA in reversing ethanol-mediated decrease in HDL-ApoE. Our previous work [58] also demonstrated that HDL from low-3FA-fed animals were more efficient in carrying out reverse cholesterol transport (RCT) function compared to the control animals regardless of whether the animals were on alcohol or control diet. We found [62] that cholesterol uptake by Hep-G2 cells from reconstituted HDL was stimulated by sphingomyelin (SPM). HDL phospholipid acyl chain composition is known to influence cholesterol efflux [63]. We also showed that chronic ethanol preferentially decreased SPM concentration in HDL of alcoholics leading to its impaired RCT function [64].

The present study shows that SP downregulated ethanolmediated overexpression of PGC1 $\beta$, SREBP-1, and its target lipogenic genes such as ACC (Figure 2), whereas it restored ethanol-mediated downregulation of SIRT1, PGC1 $\alpha$, and lipid oxidizing genes such as CPT1 (Figure 4). Overall, our results suggest that the relative hypolipidemic effects of SP compared to low- $\omega 3 \mathrm{FA}$ in regulating alcoholic hepatosteatosis were more due to alteration in the lipogenic pathway, whereas that of low- $\omega 3$ FA compared to SP was more due to alteration in the lipid oxidizing pathway. 
In summary, this study has demonstrated the following. (1) Low- $\omega 3$ FA and SP reduced alcoholic hyperlipidemia as well as hepatic lipid accumulation as evidenced by decreased liver cholesterol and triglycerides as well as hepatic histological lipid scores. (2) Low- $\omega 3$ FA and SP prevented alcoholmediated downregulation of SIRT1 and PGC1 $\alpha$ and their target fatty acid oxidation pathway genes. (3) Low- $\omega 3 \mathrm{FA}$ and SP attenuated alcohol-mediated upregulation of PGC1 $\beta$, SREBP1c, and its target lipogenic pathway genes. (4) Low$\omega 3 \mathrm{FA}$ and SP decreased the liver nuclear SREBP1c level that was increased by chronic ethanol treatment. (5) Low$\omega 3 \mathrm{FA}$ and SP restored the hepatic level of pAMPK that was decreased by chronic alcohol treatment.

\section{Conclusion}

Unlike high dietary $\omega 3 \mathrm{FA}$, low dietary $\omega 3 \mathrm{FA}$ protects against chronic alcohol-induced liver injury. We have demonstrated that low- $\omega 3 \mathrm{FA}$ and SP could potentially upregulate SIRT1/PGC1 $\alpha$ and downregulate PGC1 $\beta /$ SREBP1c signaling pathways in alleviating alcoholic hepatosteatosis and liver injury. Thus, our study opens this field to explore other new therapeutic agents targeted on $\mathrm{PGCl} \alpha$ and $\mathrm{PGCl} \beta$ pathways for protection against not only alcoholic liver diseases but also metabolic syndrome and obesity, the major world-wide health problems, especially when superimposed in alcohol abusers.

\section{Competing Interests}

The authors declare that they have no competing interests.

\section{Acknowledgments}

This work was supported by NIH Grant R01AA0207020 (PI:MRL).

\section{References}

[1] C. E. Cojocariu, A. V. Trifan, I. Gîrleanu, and C. Stanciu, "Alcoholic liver diseas-epidemiology and risk factors," Revista medico-chirurgicala a Societatii de Medici si Naturalisti din Iasi, vol. 118, no. 4, pp. 910-917, 2014.

[2] J.-Y. Zhou, Z.-A. Jiang, C.-Y. Zhao, Z. Zhen, W. Wang, and A. A. Nanji, "Long-term binge and escalating ethanol exposure causes necroinflammation and fibrosis in rat liver," Alcoholism: Clinical and Experimental Research, vol. 37, no. 2, pp. 213-222, 2013.

[3] T. Zeng and K.-Q. Xie, "Ethanol and liver: recent advances in the mechanisms of ethanol-induced hepatosteatosis," Archives of Toxicology, vol. 83, no. 12, pp. 1075-1081, 2009.

[4] M. R. Lakshman, "Some novel insights into the pathogenesis of alcoholic steatosis," Alcohol, vol. 34, no. 1, pp. 45-48, 2004.

[5] E. E. Mulvihill and M. W. Huff, "Protection from metabolic dysregulation, obesity, and atherosclerosis by citrus flavonoids: activation of hepatic PGCl $\alpha$-mediated fatty acid oxidation," PPAR Research, vol. 2012, Article ID 857142, 9 pages, 2012.

[6] Y. Nagai, S. Yonemitsu, D. M. Erion et al., "The role of peroxisome proliferator-activated receptor $\gamma$ coactivator- $1 \beta$ in the pathogenesis of fructose-induced insulin resistance," Cell Metabolism, vol. 9, no. 3, pp. 252-264, 2009.

[7] K. Tachibana, Y. Kobayashi, T. Tanaka et al., "Gene expression profiling of potential peroxisome proliferator-activated receptor (PPAR) target genes in human hepatoblastoma cell lines inducibly expressing different PPAR isoforms," Nuclear Receptor, vol. 3, article 3, 2005.

[8] E. E. Girroir, H. E. Hollingshead, P. He, B. Zhu, G. H. Perdew, and J. M. Peters, "Quantitative expression patterns of peroxisome proliferator-activated receptor-beta/delta $(\operatorname{PPAR} \beta / \Delta)$ protein in mice," Biochemical and Biophysical Research Communications, vol. 371, no. 3, pp. 456-461, 2008.

[9] T. Varga, Z. Czimmerer, and L. Nagy, "PPARs are a unique set of fatty acid regulated transcription factors controlling both lipid metabolism and inflammation," Biochimica et Biophysica ActaMolecular Basis of Disease, vol. 1812, no. 8, pp. 1007-1022, 2011.

[10] J. M. Peters, J. E. Foreman, and F. J. Gonzalez, "Dissecting the role of peroxisome proliferator-activated receptor- $\beta / \delta$ $(\operatorname{PPAR} \beta / \delta)$ in colon, breast, and lung carcinogenesis," Cancer and Metastasis Reviews, vol. 30, no. 3-4, pp. 619-640, 2011.

[11] B. M. Spiegelman, "Transcriptional control of mitochondrial energy metabolism through the PGC1 coactivators," Novartis Foundation Symposium, vol. 287, pp. 60-69, 2007.

[12] S. Nemoto, M. M. Fergusson, and T. Finkel, "SIRT1 functionally interacts with the metabolic regulator and transcriptional coactivator PGC-1 $\alpha$," Journal of Biological Chemistry, vol. 280, no. 16, pp. 16456-16460, 2005.

[13] Y. Li, S. Xu, M. M. Mihaylova et al., "AMPK phosphorylates and inhibits SREBP activity to attenuate hepatic steatosis and atherosclerosis in diet-induced insulin-resistant mice," Cell Metabolism, vol. 13, no. 4, pp. 376-388, 2011.

[14] S. Carradori, D. Secci, and A. Mai, "Epigenetic modulation of PGC-1 $\alpha$ activity by GCN5 inhibitors: WO2010007085," Expert Opinion on Therapeutic Patents, vol. 21, no. 10, pp. 1651-1656, 2011.

[15] M. Lagouge, C. Argmann, Z. Gerhart-Hines et al., "Resveratrol improves mitochondrial function and protects against metabolic disease by activating SIRT1 and PGC-1 $\alpha$," Cell, vol. 127, no. 6, pp. 1109-1122, 2006.

[16] M. You, Q. Cao, X. Liang, J. M. Ajmo, and G. C. Ness, "Mammalian sirtuin 1 is involved in the protective action of dietary saturated fat against alcoholic fatty liver in mice," Journal of Nutrition, vol. 138, no. 3, pp. 497-501, 2008.

[17] J. B. Kim and B. M. Spiegelman, "ADD1/SREBP1 promotes adipocyte differentiation and gene expression linked to fatty acid metabolism," Genes and Development, vol. 10, no. 9, pp. 1096-1107, 1996.

[18] H. Shimano, J. D. Horton, R. E. Hammer, I. Shimomura, M. S. Brown, and J. L. Goldstein, "Overproduction of cholesterol and fatty acids causes massive liver enlargement in transgenic mice expressing truncated SREBP-1a," Journal of Clinical Investigation, vol. 98, no. 7, pp. 1575-1584, 1996.

[19] M. Burckhardt, M. Herke, T. Wustmann, S. Watzke, G. Langer, and A. Fink, "Omega-3 fatty acids for the treatment of dementia," The Cochrane Database of Systematic Reviews, vol. 4, Article ID CD009002, 2016.

[20] K. S. Montgomery, "Soy protein," The Journal of Perinatal Education, vol. 12, no. 3, pp. 42-45, 2003.

[21] W. X. Chao, "Health effects of soy protein and isoflavones in humans," Journal of Nutrition, vol. 138, no. 6, pp. 1244S-1249S, 2008. 
[22] D. Cutuli, "Functional and structural benefits induced by omega-3 polyunsaturated fatty acids during aging," Current Neuropharmacology, vol. 14, no. 8, 2016.

[23] B. E. Phillipson, D. W. Rothrock, W. E. Connor, W. S. Harris, and D. R. Illingworth, "Reduction of plasma lipids, lipoproteins, and apoproteins by dietary fish oils in patients with hypertriglyceridemia," The New England Journal of Medicine, vol. 312, no. 19, pp. 1210-1216, 1985.

[24] S.-C. Yang, S.-M. Liu, H.-Y. Yang, Y.-H. Lin, and J.-R. Chen, "Soybean protein hydrolysate improves plasma and liver lipid profiles in rats fed high-cholesterol diet," Journal of the American College of Nutrition, vol. 26, no. 5, pp. 416-423, 2007.

[25] T. Hashidume, T. Sasaki, J. Inoue, and R. Sato, "Consumption of soy protein isolate reduces hepatic SREBP-1c and lipogenic gene expression in wild-type mice, but not in FXR-deficient mice," Bioscience, Biotechnology and Biochemistry, vol. 75, no. 9, pp. 1702-1707, 2011.

[26] M. J. Ronis, Y. Chen, J. Badeaux, and T. M. Badger, "Dietary soy protein isolate attenuates metabolic syndrome in rats via effects on PPAR, LXR, and SREBP signaling," The Journal of Nutrition, vol. 139, no. 8, pp. 1431-1438, 2009.

[27] A. R. Tovar, I. Torre-Villalvazo, M. Ochoa et al., "Soy protein reduces hepatic lipotoxicity in hyperinsulinemic obese Zucker fa/fa rats," Journal of Lipid Research, vol. 46, no. 9, pp. 1823-1832, 2005.

[28] C. S. Lieber and L. M. DeCarli, "The feeding of alcohol in liquid diets: two decades of applications and 1982 update," Alcoholism: Clinical and Experimental Research, vol. 6, no. 4, pp. 523-531, 1982.

[29] P. G. Reeves, F. H. Nielsen, and G. C. Fahey Jr., "AIN-93 purified diets for laboratory rodents: final report of the American Institute of Nutrition ad hoc writing committee on the reformulation of the AIN-76A rodent diet," The Journal of Nutrition, vol. 123, no. 11, pp. 1939-1951, 1993.

[30] L. I. Gidez, G. J. Miller, M. Burstein, S. Slagle, and H. A. Eder, "Separation and quantitation of subclasses of human plasma high density lipoproteins by a simple precipitation procedure," Journal of Lipid Research, vol. 23, no. 8, pp. 1206-1223, 1982.

[31] M. Burstein, H. R. Scholnick, and R. Morfin, "Rapid method for the isolation of lipoproteins from human serum by precipitation with polyanions," Journal of Lipid Research, vol. 11, no. 6, pp. 583-595, 1970.

[32] C. C. Allain, L. S. Poon, and C. S. G. Chan, "Enzymatic determination of total serum cholesterol," Clinical Chemistry, vol. 20, no. 4, pp. 470-475, 1974.

[33] M. W. McGowan, J. D. Artiss, D. R. Strandbergh, and B. Zak, "A peroxidase-coupled method for the colorimetric determination of serum triglycerides," Clinical Chemistry, vol. 29, no. 3, pp. 538-542, 1983.

[34] M. M. Bradford, "A rapid and sensitive method for the quantitation of microgram quantities of protein utilizing the principle of protein-dye binding," Analytical Biochemistry, vol. 72, no. 1-2, pp. 248-254, 1976.

[35] A. Zlatkis and B. Zak, "Study of a new cholesterol reagent," Analytical Biochemistry, vol. 29, no. 1, pp. 143-148, 1969.

[36] S. K. Basu, J. L. Goldstein, G. W. Anderson, and M. S. Brown, "Degradation of cationized low density lipoprotein and regulation of cholesterol metabolism in homozygous familial hypercholesterolemia fibroblasts," Proceedings of the National Academy of Sciences of the United States of America, vol. 73, no. 9, pp. 3178-3182, 1976.
[37] R. Varatharajalu, M. Garige, L. C. Leckey et al., "Adverse signaling of scavenger receptor class B1 and PGC1s in alcoholic hepatosteatosis and steatohepatitis and protection by betaine in rat," The American Journal of Pathology, vol. 184, no. 7, pp. 20352044, 2014.

[38] J. T. Rodgers, C. Lerin, W. Haas, S. P. Gygi, B. M. Spiegelman, and P. Puigserver, "Nutrient control of glucose homeostasis through a complex of PGC-1 $\alpha$ and SIRT1," Nature, vol. 434, no. 7029, pp. 113-118, 2005.

[39] M. Rakhshandehroo, S. Kersten, B. Knoch, and M. Müller, "Peroxisome proliferator-activated receptor alpha target genes," PPAR Research, vol. 2010, Article ID 612089, 20 pages, 2010.

[40] A. Satoh, L. Stein, and S. Imai, "The role of mammalian sirtuins in the regulation of metabolism, aging, and longevity," Handbook of Experimental Pharmacology, vol. 206, pp. 125-162, 2011.

[41] C. S. Lieber, M. A. Leo, X. Wang, and L. M. DeCarli, "Effect of chronic alcohol consumption on Hepatic SIRT1 and PGC- $1 \alpha$ in rats," Biochemical and Biophysical Research Communications, vol. 370, no. 1, pp. 44-48, 2008.

[42] M. Fischer, M. You, M. Matsumoto, and D. W. Crabb, "Peroxisome proliferator-activated receptor $\alpha(\operatorname{PPAR} \alpha)$ agonist treatment reverses PPAR $\alpha$ dysfunction and abnormalities in hepatic lipid metabolism in ethanol-fed mice," The Journal of Biological Chemistry, vol. 278, no. 30, pp. 27997-28004, 2003.

[43] N. Enomoto, Y. Takei, M. Hirose et al., "Prevention of ethanolinduced liver injury in rats by an agonist of peroxisome proliferator-activated receptor- $\gamma$, pioglitazone," Journal of Pharmacology and Experimental Therapeutics, vol. 306, no. 3, pp. 846-854, 2003.

[44] K. Tomita, T. Azuma, N. Kitamura et al., "Pioglitazone prevents alcohol-induced fatty liver in rats through up-regulation of cMet," Gastroenterology, vol. 126, no. 3, pp. 873-885, 2004.

[45] A. A. Nanji, S. M. Hossein Sadrzadeh, E. K. Yang, F. Fogt, M. Meydani, and A. J. Dannenberg, "Dietary saturated fatty acids: a novel treatment for alcoholic liver disease," Gastroenterology, vol. 109, no. 2, pp. 547-554, 1995.

[46] M. J. J. Ronis, S. Korourian, M. Zipperman, R. Hakkak, and T. M. Badger, "Dietary saturated fat reduces alcoholic hepatotoxicity in rats by altering fatty acid metabolism and membrane composition," Journal of Nutrition, vol. 134, no. 4, pp. 904-912, 2004.

[47] B.-J. Song, K.-H. Moon, N. U. Olsson, and N. Salem Jr., "Prevention of alcoholic fatty liver and mitochondrial dysfunction in the rat by long-chain polyunsaturated fatty acids," Journal of Hepatology, vol. 49, no. 2, pp. 262-273, 2008.

[48] L.-L. Huang, J.-B. Wan, B. Wang et al., "Suppression of acute ethanol-induced hepatic steatosis by docosahexaenoic acid is associated with downregulation of stearoyl-CoA desaturase 1 and inflammatory cytokines," Prostaglandins, Leukotrienes and Essential Fatty Acids, vol. 88, no. 5, pp. 347-353, 2013.

[49] S. Wada, T. Yamazaki, Y. Kawano, S. Miura, and O. Ezaki, "Fish oil fed prior to ethanol administration prevents acute ethanolinduced fatty liver in mice," Journal of Hepatology, vol. 49, no. 3, pp. 441-450, 2008.

[50] M. You, M. Fischer, M. A. Deeg, and D. W. Crabb, "Ethanol induces fatty acid synthesis pathways by activation of sterol regulatory element-binding protein (SREBP)," The Journal of Biological Chemistry, vol. 277, no. 32, pp. 29342-29347, 2002.

[51] S. H. Ki, J. H. Choi, C. W. Kim, and S. G. Kim, "Combined metadoxine and garlic oil treatment efficaciously abrogates 
alcoholic steatosis and CYP2E1 induction in rat liver with restoration of AMPK activity," Chemico-Biological Interactions, vol. 169, no. 2, pp. 80-90, 2007.

[52] Y. Lu, J. Zhuge, X. Wang, J. Bai, and A. I. Cederbaum, "Cytochrome P450 2E1 contributes to ethanol-induced fatty liver in mice," Hepatology, vol. 47, no. 5, pp. 1483-1494, 2008.

[53] T. Zeng, C.-L. Zhang, F.-Y. Song, X.-L. Zhao, and K.-Q. $\mathrm{Xie}$ " $\mathrm{CMZ}$ reversed chronic ethanol-induced disturbance of PPAR- $\alpha$ possibly by suppressing oxidative stress and PGC- $1 \alpha$ acetylation, and activating the MAPK and GSK3 $\beta$ pathway," PLoS ONE, vol. 9, no. 6, Article ID e98658, 2014.

[54] H. Tsukamoto, S. J. Towner, L. M. Ciofalo, and S. W. French, "Ethanol-induced liver fibrosis in rats fed high fat diet," Нераtology, vol. 6, no. 5, pp. 814-822, 1986.

[55] S. W. French, "Nutrition in the pathogenesis of alcoholic liver disease," Alcohol and Alcoholism, vol. 28, no. 1, pp. 97-109, 1993.

[56] A. A. Nanji, C. L. Mendenhall, and S. W. French, "Beef fat prevents alcoholic liver disease in the rat," Alcoholism: Clinical and Experimental Research, vol. 13, no. 1, pp. 15-19, 1989.

[57] M. R. Lakshman, S. J. Chirtel, and L. L. Chambers, "Roles of $\omega 3$ fatty acids and chronic ethanol in the regulation of plasma and liver lipids and plasma apoproteins A1 and E in rats," Journal of Nutrition, vol. 118, no. 11, pp. 1299-1303, 1988.

[58] P. Marmillot, M. N. Rao, Q.-H. Liu, S. J. Chirtel, and M. R. Lakshman, "Effect of dietary omega-3 fatty acids and chronic ethanol consumption on reverse cholesterol transport in rats," Metabolism: Clinical and Experimental, vol. 49, no. 4, pp. 508$512,2000$.

[59] M. N. Rao, Q.-H. Liu, P. Marmillot, L. B. Seeff, D. B. Strader, and M. R. Lakshman, "High-density lipoproteins from human alcoholics exhibit impaired reverse cholesterol transport function," Metabolism: Clinical and Experimental, vol. 49, no. 11, pp. 1406-1410, 2000.

[60] C. S. Lieber, L. M. DeCarli, K. M. Mak, C.-I. Kim, and M. A. Leo, "Attenuation of alcohol-induced hepatic fibrosis by polyunsaturated lecithin," Hepatology, vol. 12, no. 6, pp. 13901398, 1990.

[61] C. S. Lieber, S. J. Robins, L. Jianjun et al., "Phosphatidylcholine protects against fibrosis and cirrhosis in the baboon," Gastroenterology, vol. 106, no. 1, pp. 152-159, 1994.

[62] P. Marmillot, S. Patel, and M. R. Lakshman, "Reverse cholesterol transport is regulated by varying fatty acyl chain saturation and sphingomyelin content in reconstituted high-density lipoproteins," Metabolism: Clinical and Experimental, vol. 56, no. 2, pp. 251-259, 2007.

[63] W. S. Davidson, K. L. Gillotte, S. Lund-Katz, W. J. Johnson, G. H. Rothblat, and M. C. Phillips, "The effect of high density lipoprotein phospholipid acyl chain composition on the efflux of cellular free cholesterol," The Journal of Biological Chemistry, vol. 270, no. 11, pp. 5882-5890, 1995.

[64] P. Marmillot, J. Munoz, S. Patel, M. Garige, R. B. Rosse, and M. R. Lakshman, "Long-term ethanol consumption impairs reverse cholesterol transport function of high-density lipoproteins by depleting high-density lipoprotein sphingomyelin both in rats and in humans," Metabolism: Clinical and Experimental, vol. 56, no. 7, pp. 947-953, 2007. 


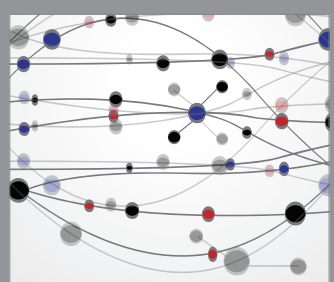

The Scientific World Journal
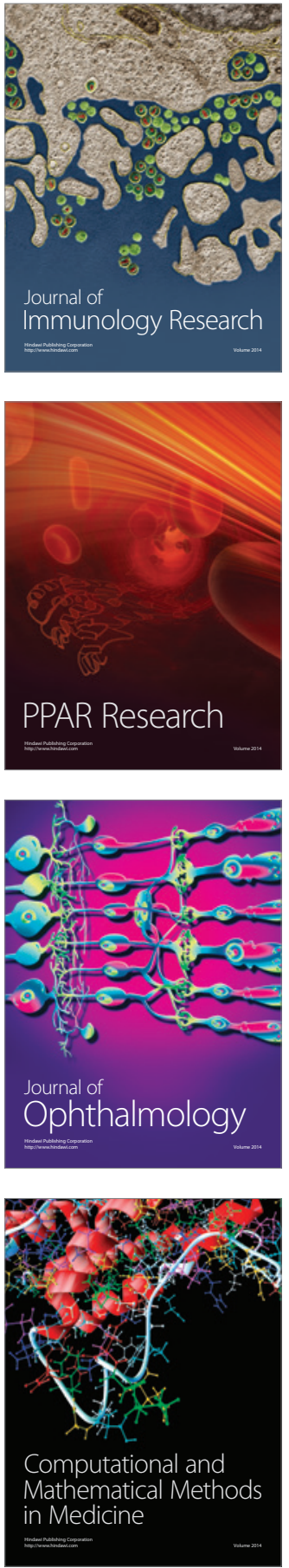

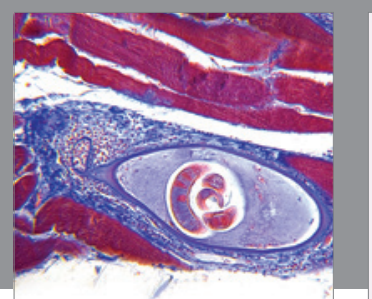

Gastroenterology Research and Practice

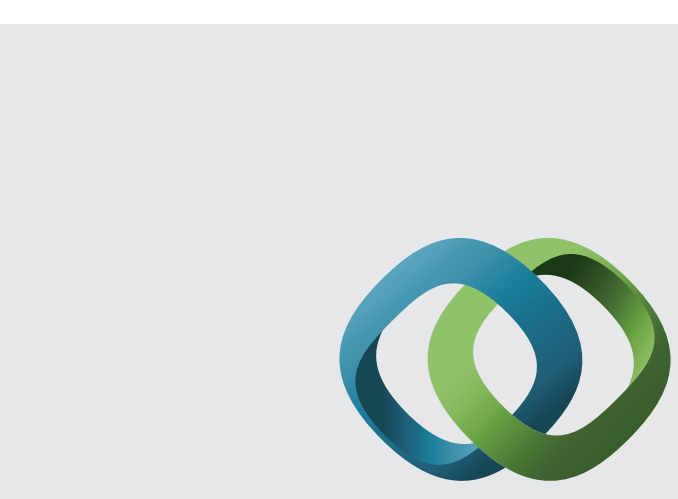

\section{Hindawi}

Submit your manuscripts at

http://www.hindawi.com
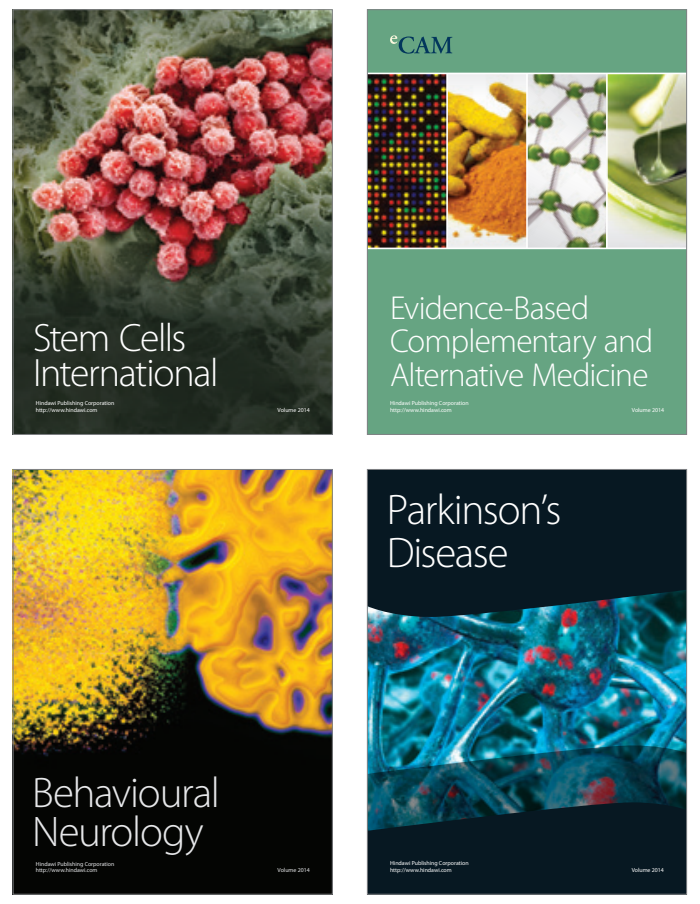
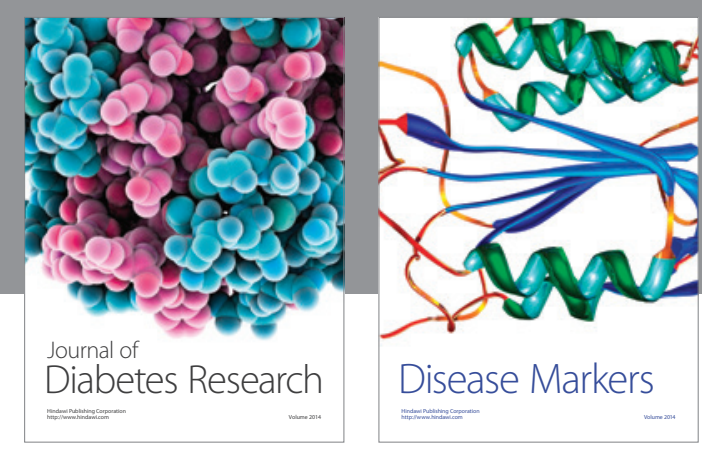

Disease Markers
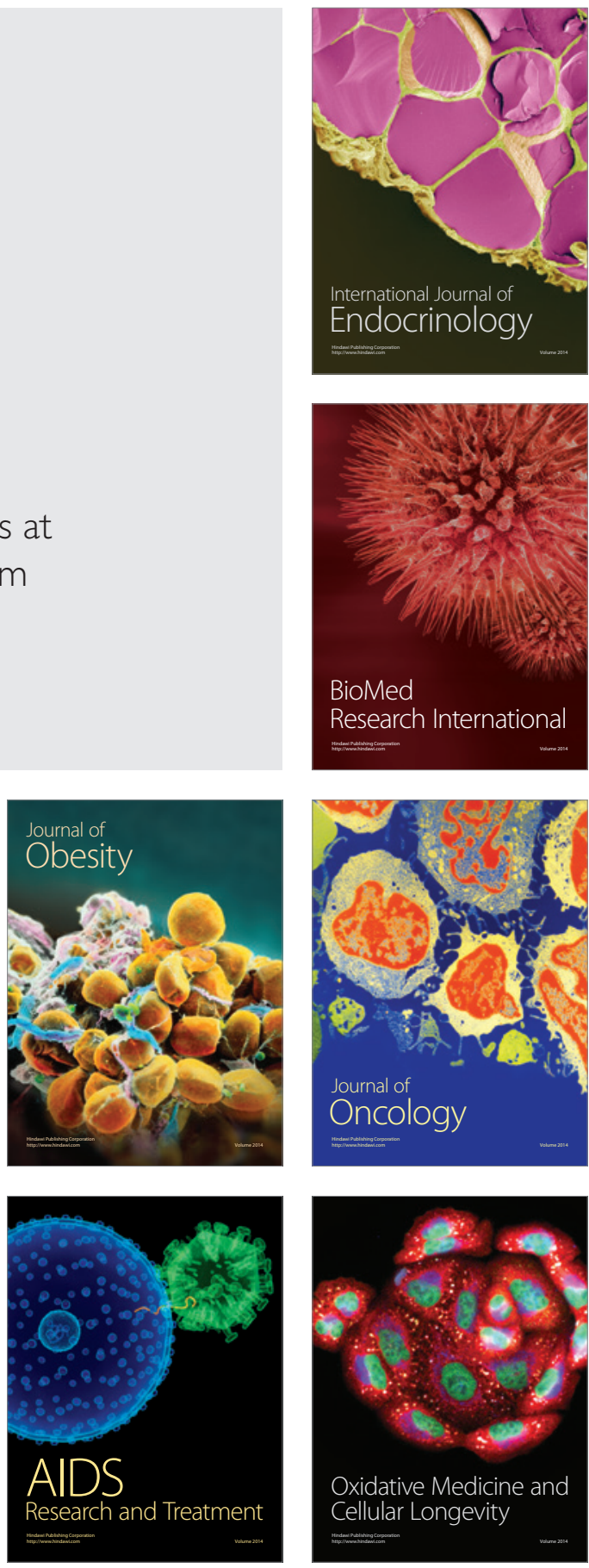